\title{
Feedback control of quantum entanglement in a two-spin system
}

\author{
Naoki Yamamoto, Koji Tsumura and Shinji Hara
}

\begin{abstract}
A pair of spins is the most simple quantum system that can possess entanglement, a non-classical property playing an essential role in quantum information technologies. In this paper, feedback control problems of the two-spin system conditioned on a continuous measurement are considered. In order to make some useful formulas in stochastic control theory directly applicable, we first derive a two-dimensional description of the system. We then prove that a feedback controller stabilizes an entangled state of the two spins almost globally with probability one. Furthermore, it is shown that another entangled state, which corresponds to a non-equilibrium point of the dynamics, is stabilized via feedback in the sense that the expectation of the distance from the target can be made arbitrarily small.
\end{abstract}

\section{INTRODUCTION}

The methodologies to manipulate quantum systems have been rapidly progressed owing to the development of the quantum filtering theory [1] based on the scheme of a continuous measurement [2], which realizes feedback control of quantum systems. A quantum state subject to a perfect continuous measurement obeys a nonlinear stochastic differential equation called the stochastic Schrödinger equation (SSE) [3], [4], and many important issues in quantum theory have been formulated as control problems of the SSE and solved. For example, stabilization of a single-spin system under the noisy environment [5] and continuous quantum error-correction via feedback control [6].

The above-mentioned feedback control scheme also has shed a new light on many important subjects associated with entanglement. The entanglement is a specific property that represents a correlation between quantum states, and it can be used to construct non-classical information processing, e.g., quantum computations [7], [8] and the quantum teleportation [9]. Now we consider a typical control problem of entanglement; for a spin system, which is the most major finitedimensional quantum system, stabilize an entangled state corresponding to the equilibrium points of the SSE. Stockton [10] has considered a symmetric multi-spin system and developed two general feedback controllers; however, it was numerically shown that they do not achieve the deterministic convergence into target entangled states. Nevertheless, owing to the investigations in [10], van Handel [11] has found a feedback controller stabilizing an equilibrium point globally and deterministically for the single-spin system, although in

N. Yamamoto is with Control and Dynamical Systems, California Institute of Technology, Pasadena, California 91125, USA naoki@cds.caltech. edu

K. Tsumura and S. Hara are with the Department of Information Physics and Computing, Graduate School of Information Science and Technology, University of Tokyo, Hongo 7-31, Bunkyo-ku, Tokyo 113-0033, Japan \{Koji_Tsumura, Shinji_Hara\}@ipc.i.u-tokyo.ac.jp this case the system is composed of only one spin and does not possess entanglement.

Therefore in this paper, we consider a two-spin system as the first step toward the perfect stabilization of the entangled state in the multi-spin system. We should note that the twospin system itself is a fundamental element to create the highly-entangled states. Actually, in quantum computing all the (unitary) entangle operations on arbitrarily many spins can be expressed as compositions of those on the two spins, which is known as universality of quantum circuit [8], [12]. Hence the stabilization of the entangled state in the two-spin system is of great significance for the design of several kinds of quantum devices.

The contributions of this paper are the following. (i) Under an assumption, we derive a two-dimensional description of the SSE, which can make some useful formulas in stochastic control theory directly applicable. (ii) We exploit a controller which attains almost global convergence into an entangled state of the two spins with probability one. (iii) The controller given in (ii) cannot change the degree of the entanglement. Therefore we propose a stabilizing controller of a non-equilibrium state with maximal degree of entanglement in the following sense: the controller can decreases arbitrarily the mean value of a positive function which can be regarded as the distance from the target.

\section{PRELIMINARIES}

\section{A. Quantum state}

The quantum state of an $n$-dimensional system is represented by a complex vector $\phi \in \mathbf{C}^{n}$ such that $\phi$ and $-\phi$ are identified, and $\|\phi\|:=\sqrt{\phi^{*} \phi}=1$ holds $^{1}$. Here the asterisk $*$ denotes the complex conjugate. Roughly speaking, the dimension of the quantum state corresponds to the number of possible ways in which the associated classical phenomenon occurs. For example, for a single-spin system there are two possible ways: the spin-up and the spin-down states along an axis (we here call it the $z$-axis). That is, the dimension of the single-spin system is two. A typical selection of the two vectors representing up and down states are respectively taken as

$$
\phi_{\mathrm{u}}=\left[\begin{array}{ll}
1 & 0
\end{array}\right]^{T}, \quad \phi_{\mathrm{d}}=\left[\begin{array}{ll}
0 & 1
\end{array}\right]^{T} .
$$

An important fact is that the system can take any superposition of states, which is called the superposition principle in quantum theory. For the single-spin system, the superposition

\footnotetext{
${ }^{1}$ Through this paper, we consider so-called pure states. If there is a lack of information about the quantum state, it must be represented by a positive semidefi nite matrix $P \geq 0$ satisfying $\operatorname{tr} P=1$, instead of a vector $\boldsymbol{x}$. This is called a mixed state.
} 
of $\phi_{\mathrm{u}}$ and $\phi_{\mathrm{d}}$ is of the form $\phi=a \phi_{\mathrm{u}}+b \phi_{\mathrm{d}}=[a b]^{T}$, where the complex coefficients $a, b \in \mathbf{C}$ satisfy $|a|^{2}+|b|^{2}=1$. For instance, the quantum state $\phi_{\mathrm{x}}=(1 / \sqrt{2})\left[\begin{array}{ll}1 & 1\end{array}\right]^{T}$ represents a spin state pointing the $x$-axis.

In this paper, we focus on a system composed of two spins. The two-spin system is described in the product space of the single-spin, i.e., $\mathbf{C}^{4}=\mathbf{C}^{2} \otimes \mathbf{C}^{2}$. The four basis can be taken as up-up, up-down, down-up and down-down states. For example, the up-up state is given in $\mathbf{C}^{4}$ by

$$
\phi_{\mathrm{u}} \otimes \phi_{\mathrm{u}}=\left[\begin{array}{l}
1 \\
0
\end{array}\right] \otimes\left[\begin{array}{l}
1 \\
0
\end{array}\right]=\left[\begin{array}{llll}
1 & 0 & 0 & 0
\end{array}\right]^{T}
$$

where $\otimes$ denotes Kronecker's product. By the analogous way, we obtain four basis as follows:

$$
\begin{aligned}
& \left\{\phi_{\mathrm{u}} \otimes \phi_{\mathrm{u}}, \phi_{\mathrm{u}} \otimes \phi_{\mathrm{d}}, \phi_{\mathrm{d}} \otimes \phi_{\mathrm{u}}, \phi_{\mathrm{d}} \otimes \phi_{\mathrm{d}}\right\} \\
& \quad=\left\{\left[\begin{array}{l}
1 \\
0 \\
0 \\
0
\end{array}\right],\left[\begin{array}{l}
0 \\
1 \\
0 \\
0
\end{array}\right],\left[\begin{array}{l}
0 \\
0 \\
1 \\
0
\end{array}\right],\left[\begin{array}{l}
0 \\
0 \\
0 \\
1
\end{array}\right]\right\} .
\end{aligned}
$$

Any quantum state of the two-spin system is described by a superposition of the above four basis, i.e., $\phi=$ $\left[\begin{array}{llll}a_{1} & a_{2} & a_{3} & a_{4}\end{array}\right]^{T}\left(\sum_{i}\left|a_{i}\right|^{2}=1\right)$. We here mention about a non-classical correlation between the spins. When the twospin state takes a special form:

$$
\phi=\phi_{1} \otimes \phi_{2}=\left[\begin{array}{l}
a \\
b
\end{array}\right] \otimes\left[\begin{array}{l}
c \\
d
\end{array}\right]=\left[\begin{array}{llll}
a c & a d & b c & b d
\end{array}\right]^{T},
$$

there is no correlation between the spins. On the other hand, a quantum state that cannot be expressed by a product state as shown in Eq. (1) has a specific quantum correlation called entanglement [8]. For example, an entangled state

$$
\phi=a \phi_{\mathrm{u}} \otimes \phi_{\mathrm{d}}+b \phi_{\mathrm{d}} \otimes \phi_{\mathrm{u}}=\left[\begin{array}{llll}
0 & a & b & 0
\end{array}\right]^{T}, a \neq 0, b \neq 0
$$

cannot be written by a product form $\phi=\phi_{1} \otimes \phi_{2}$. In particular, there are some quantum states whose entanglement has the maximum degree in which case many quantum information processing achieve their highest performances. This paper focuses on the following two maximally entangled states [8]:

$$
\begin{aligned}
& \boldsymbol{\Psi}=\frac{1}{\sqrt{2}}\left(\boldsymbol{x}_{\mathrm{u}-\mathrm{d}}+\boldsymbol{x}_{\mathrm{d}-\mathrm{u}}\right)=\frac{1}{\sqrt{2}}\left[\begin{array}{llll}
0 & 1 & 1 & 0
\end{array}\right]^{T}, \\
& \boldsymbol{\Phi}=\frac{1}{\sqrt{2}}\left(\boldsymbol{x}_{\mathrm{u}-\mathrm{u}}+\boldsymbol{x}_{\mathrm{d}-\mathrm{d}}\right)=\frac{1}{\sqrt{2}}\left[\begin{array}{llll}
1 & 0 & 0 & 1
\end{array}\right]^{T} .
\end{aligned}
$$

\section{B. The stochastic Schrödinger equation}

The SSE describes the time-evolution of a quantum system subject to a continuous measurement. In quantum theory, a physical property that in principle can be measured is represented by a self-adjoint operator, especially an Hermite matrix when considering finite-dimensional systems. Now we focus on an open system in contact with the electromagnetic field; then, measuring the field continuously and obtaining information on the system, we can keep track the best estimate of the system's quantum state. When the measurement is perfect, the estimated quantum state $\boldsymbol{x}(t)$ obeys the SSE of the form;

$$
\begin{aligned}
& d \boldsymbol{x}=\left[-\mathrm{i} u(t) H-\frac{1}{2}(C-\langle C\rangle I)^{2}\right] \boldsymbol{x} d t \\
& +(d y-\langle C\rangle d t)(C-\langle C\rangle I) \boldsymbol{x},
\end{aligned}
$$

where $C$ is the measured observable associated with the system. We here defined $\langle C\rangle:=\boldsymbol{x}^{*} C \boldsymbol{x}$. Also, $u(t) \in \mathbf{R}$ denotes the control input, and $H$ is an Hermite matrix called the Hamiltonian. The measurement data $y(t) \in \mathbf{R}$ is associated with the standard Wiener process [13];

$$
d y-\langle C\rangle d t=d W, \quad \mathbf{E}[d W(t)]=0, \mathbf{E}\left[d W(t)^{2}\right]=d t,
$$

where $\mathbf{E}$ denotes the expectation. Hence if we know the initial value $\boldsymbol{x}(0)$, updating the filtering equation (5) with the data $y(t)$, we can obtain a perfect knowledge about the time-dependent series of $\boldsymbol{x}(t)$, which eventually enables us to control the quantum system via state-feedback. That is, the objective is to control the SSE of the form

$d \boldsymbol{x}=\left[-\mathrm{i} u(t) H-\frac{1}{2}(C-\langle C\rangle I)^{2}\right] \boldsymbol{x} d t+(C-\langle C\rangle I) \boldsymbol{x} d W$

with the state-dependent feedback law $u(t)=u(\boldsymbol{x}(t))$.

It is readily seen that the variable $\boldsymbol{x}(t)$ obeying the SSE satisfies $d\|\boldsymbol{x}\|^{2}=2\langle C\rangle\left(1-\|\boldsymbol{x}\|^{2}\right) d W$, which implies that the condition of quantum states always holds: $\|\boldsymbol{x}(t)\|=1 \forall t \geq 0$ if $\|\boldsymbol{x}(0)\|=1$. Also, the stochastic evolution (6) has an important property as follows. Let us consider a non-negative function $V(\boldsymbol{x})=\left\langle C^{2}\right\rangle-\langle C\rangle^{2}=\boldsymbol{x}^{*} C^{2} \boldsymbol{x}-\left(\boldsymbol{x}^{*} C \boldsymbol{x}\right)^{2}$, which becomes zero if and only if $\boldsymbol{x}$ is an eigenvector of $C$. When $u(t)=0$, a lengthy calculation yields $\mathcal{L} V=-4 V^{2} \leq 0$. Hence from Lemma 2 in Appendix, we have $\lim _{t \rightarrow \infty} \mathcal{L} V=$ 0 . This means that $\boldsymbol{x}(\infty)$ must be one of the eigenvectors of $C$. This phenomenon is called quantum state reduction, and a numerical simulation provided in the next section will show us an actual stochastic evolution of the state reduction.

\section{THE STOCHASTIC DYNAMICS OF THE TWO-SPIN SYSTEM}

Let us firstly explain the standard measurement process of a single-spin in terms of the Stern-Gerlach device [14]. The Stern-Gerlach device consists of a particle beam traveling a region with a spatially non-uniform transverse magnetic field. The effect of this non-uniformity causes a transverse magnetic force on the particle, which will depend on the particle's magnetic moment. When the device is set up to measure the $\{x, y, z\}$-axis of the (single) particle, the corresponding quantum observables are respectively represented by the Pauli matrices;

$$
\sigma_{x}=\left[\begin{array}{ll}
0 & 1 \\
1 & 0
\end{array}\right], \sigma_{y}=\left[\begin{array}{cc}
0 & -\mathrm{i} \\
\mathrm{i} & 0
\end{array}\right], \sigma_{z}=\left[\begin{array}{cc}
1 & 0 \\
0 & -1
\end{array}\right] .
$$

Note that both $\boldsymbol{x}_{\mathrm{up}}$ and $\boldsymbol{x}_{\text {down }}$ are an eigenvector of $\sigma_{z}$.

We now measure the two spins along each $z$-axis simultaneously, which correspond to the measurement of an observable

$$
C=\frac{\sqrt{\gamma}}{2}\left(\sigma_{z} \otimes I+I \otimes \sigma_{z}\right)=\sqrt{\gamma} \operatorname{diag}\{1,0,0,-1\},
$$


where $I=\operatorname{diag}\{1,1\}$ and the positive parameter $\gamma$ represents the measurement strength. Without the control term, the SSE describing the two-spin system turns out to be

$$
\begin{aligned}
d\left[\begin{array}{l}
x_{1} \\
x_{2} \\
x_{3} \\
x_{4}
\end{array}\right]=\frac{1}{2}\left[\begin{array}{c}
-(\sqrt{\gamma}-\langle C\rangle)^{2} x_{1} \\
-\langle C\rangle^{2} x_{2} \\
-\langle C\rangle^{2} x_{3} \\
-(\sqrt{\gamma}+\langle C\rangle)^{2} x_{4}
\end{array}\right] d t \\
+\left[\begin{array}{c}
(\sqrt{\gamma}-\langle C\rangle) x_{1} \\
-\langle C\rangle x_{2} \\
-\langle C\rangle x_{3} \\
-(\sqrt{\gamma}+\langle C\rangle) x_{4}
\end{array}\right] d W .
\end{aligned}
$$

A remarkable fact is that the above dynamics has an invariant quantity $x_{2}(t) / x_{3}(t)=x_{2}(0) / x_{3}(0)$ since a relation $d\left(x_{2} / x_{3}\right)=0$ always holds. Therefore, we introduce a constant value $k$ in order to denote the ratio of the initial points $x_{2}(0)$ and $x_{3}(0)$, i.e.,

$$
k:=x_{2}(0) / x_{3}(0) \quad\left(x_{3}(0) \neq 0\right) .
$$

When $x_{3}(0)=0$, we always have $x_{3}(t)=0, \forall t>0$. Here we assume that the initial state $\boldsymbol{x}(0)$ is real;

$$
\boldsymbol{x}(0) \in \mathbf{R}^{4} .
$$

This is a practical assumption because a number of typical initial states satisfy this condition. In this case, $x_{i}(t)(i=$ $1, \ldots, 4)$ are obviously real for all $t \geq 0$, and consequently, the dynamics can be written by only two real parameters due to the invariant quantities $x_{2}(t)=k x_{3}(t)$ and $\|\boldsymbol{x}(t)\|=1$. Therefore, we parameterize the state $\boldsymbol{x}$ by

$$
\boldsymbol{x}(\theta, \phi)=\left[\begin{array}{c}
\sin (\theta / 2) \cos (\phi / 2) \\
\sqrt{k^{2} /\left(1+k^{2}\right)} \cos (\theta / 2) \\
\sqrt{1 /\left(1+k^{2}\right)} \cos (\theta / 2) \\
\sin (\theta / 2) \sin (\phi / 2)
\end{array}\right] .
$$

Through lengthy calculations we have

$$
\begin{aligned}
d \theta & =-\frac{\gamma}{2} \sin \theta \cos \theta \cos ^{2} \phi d t+\sqrt{\gamma} \sin \theta \cos \phi d W \\
d \phi & =2 \gamma \cos \theta \sin \phi \cos \phi d t-2 \sqrt{\gamma} \sin \phi d W .
\end{aligned}
$$

Now the totality of the quantum states (8) constructs the sphere $S^{2}$. Then, it follows from the identification of any antipodal points on $S^{2}$ that the range of the parameters are limited to $0 \leq \theta<2 \pi$ and $0 \leq \phi<2 \pi$. The equilibrium points of the dynamics are given by

$$
(\theta, \phi)=(0,0),(0, \pi),(\pi, 0),(\pi, \pi),
$$

which respectively correspond to

$$
\left\{\boldsymbol{\Psi}_{k}, \boldsymbol{\Psi}_{k}, \boldsymbol{x}_{\mathrm{u}-\mathrm{u}}, \boldsymbol{x}_{\mathrm{d}-\mathrm{d}}\right\},
$$

where

$$
\boldsymbol{\Psi}_{k}:=\frac{1}{\sqrt{1+k^{2}}}\left[\begin{array}{llll}
0 & k & 1 & 0
\end{array}\right]^{T} .
$$

These are just the eigenvectors of the matrix (7). Note that $\boldsymbol{\Psi}_{k}$ is an entangled state when $k \neq 0$, whereas the other equilibrium points correspond to non-entangled states.

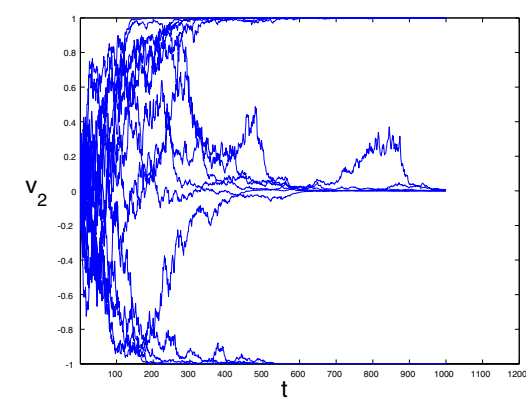

Fig. 1. Time dependence of the variable $v_{2}(\theta, \phi)$ for the autonomous SSE. The measurement strength is $\gamma=2$.

Now let us see the stochastic behavior of the autonomous dynamics (9) and (10) in a numerical simulation. As explained in the previous section, the quantum state obeying the SSE exhibits the state reduction into the eigenvectors of $C$. We consider a function

$$
v_{2}(\theta, \phi)=\frac{\langle C\rangle}{\sqrt{\gamma}}=x_{1}^{2}-x_{4}^{2}=\sin ^{2} \frac{\theta}{2} \cos \phi,
$$

where the convergences $\boldsymbol{x}(\theta, \phi) \rightarrow \boldsymbol{\Psi}_{k}, \boldsymbol{x}_{\mathrm{u}-\mathrm{u}}, \boldsymbol{x}_{\mathrm{d}-\mathrm{d}}$ respectively represent $v_{2} \rightarrow 0,+1,-1$. The initial state is set on the following non-entangled state:

$$
\boldsymbol{x}(0)=\left[\begin{array}{l}
1 / \sqrt{2} \\
1 / \sqrt{2}
\end{array}\right] \otimes\left[\begin{array}{l}
1 / \sqrt{2} \\
1 / \sqrt{2}
\end{array}\right]=\frac{1}{2}\left[\begin{array}{llll}
1 & 1 & 1 & 1
\end{array}\right]^{T},
$$

which is equivalent to $(\theta(0), \phi(0))=(\pi / 2, \pi / 2)$ and $k=$ 1. Figure 1 shows sample paths of the state reduction. In the subsequent section we aim to control this probabilistic bifurcation via feedback.

\section{FEEDBACK CONTROL OF THE TWO-SPIN SYSTEM}

\section{A. The controlled SSE}

In this paper we consider two kinds of control inputs $u_{1}(t)$ and $u_{2}(t)$ associated with the following Hamiltonians:

$$
\begin{aligned}
H_{1}= & -(1+k)\left(\sigma_{y} \otimes I+I \otimes \sigma_{y}\right) / 2 \\
& -(1-k)\left(\sigma_{y} \otimes \sigma_{z}-\sigma_{z} \otimes \sigma_{y}\right) / 2 \\
= & \mathrm{i}\left[\begin{array}{cccc}
0 & -k & -1 & 0 \\
k & 0 & 0 & -k \\
1 & 0 & 0 & -1 \\
0 & k & 1 & 0
\end{array}\right], \\
H_{2}= & -\frac{1}{2}\left(\sigma_{y} \otimes \sigma_{x}+\sigma_{x} \otimes \sigma_{y}\right)=\left[\begin{array}{cccc}
0 & 0 & 0 & \mathrm{i} \\
0 & 0 & 0 & 0 \\
0 & 0 & 0 & 0 \\
-\mathrm{i} & 0 & 0 & 0
\end{array}\right] .
\end{aligned}
$$

Recall that the constant term $k$ is determined from $x_{2}(0)=$ $k x_{3}(0)$. These Hamiltonians are physically realizable [15]. They allow us to take the same parametrization of $\boldsymbol{x}$ by $(\theta, \phi)$ discussed in the previous section since $\boldsymbol{x}$ still satisfies the relation $x_{2}(t)=k x_{3}(t)$ and $\|\boldsymbol{x}(t)\|=1$. The controlled 
SSE with the Hamiltonian $u_{1}(t) H_{1}$ is given by

$$
\begin{aligned}
d \theta & =u_{1}^{\prime}(t) \sqrt{\frac{1+k^{2}}{2}} \sin \frac{\theta}{2} \sin \left(\frac{\phi}{2}-\frac{\pi}{4}\right) d t, \\
& -\frac{\gamma}{2} \sin \theta \cos \theta \cos ^{2} \phi d t+\sqrt{\gamma} \sin \theta \cos \phi d W \\
d \phi & =u_{1}^{\prime}(t) \sqrt{\frac{1+k^{2}}{2}} \cos \frac{\theta}{2} \sin \left(\frac{\phi}{2}+\frac{\pi}{4}\right) d t \\
& +2 \gamma \cos \theta \sin \phi \cos \phi d t-2 \sqrt{\gamma} \sin \phi d W,
\end{aligned}
$$

where the input $u_{1}^{\prime}(t)$ is defined as

$$
u_{1}(t)=\frac{1}{4}\left(\sin \frac{\theta}{2}\right) u_{1}^{\prime}(t) .
$$

In the case of the second Hamiltonian $u_{2}(t) H_{2}$, the corresponding SSE is given by

$$
\begin{aligned}
& d \theta=-\frac{\gamma}{2} \sin \theta \cos \theta \cos ^{2} \phi d t+\sqrt{\gamma} \sin \theta \cos \phi d W \\
& d \phi=\left[u_{2}(t)+2 \gamma \cos \theta \sin \phi \cos \phi\right] d t-2 \sqrt{\gamma} \sin \phi d W .
\end{aligned}
$$

These controlled dynamics are completely characterized in $0 \leq \theta, \phi \leq 2 \pi$ by taking the control inputs $u_{1}^{\prime}(t)$ and $u_{2}(t)$ appropriately.

\section{B. Deterministic state preparation via feedback}

In Section III we have seen that the quantum state obeying the SSE converges into one of the eigenvectors of $C$ probabilistically. One of our objectives is to control the bifurcation of the trajectories by using the measurement data and render them deterministically and almost globally converge into a desired state, e.g., $\boldsymbol{x} \rightarrow \boldsymbol{\Psi}_{k}$. This control problem called the deterministic state preparation (DSP) has been studied for a multi-spin case [10] and for a single-spin case [11]. As mentioned in Introduction, the DSP problem has been solved so far only for the single-spin system, although it has stated in [11] that for the spin-1 system, which is regarded as the two-spin system with $k=1$, a stabilizing controller was found numerically for a fixed $\gamma$.

Hence our next subject is to solve the DSP problem for the two-spin system with arbitrary parameters $\gamma$ and $k$. We firstly provide the DSP formula of the entangled state $\Psi_{k}$ :

Theorem 1: For the controlled two-spin system (13), the feedback law

$$
u_{1}^{\prime}(t)=-M_{1} \cos \frac{\theta}{2} \sin \left(\frac{\phi}{2}-\frac{\pi}{4}\right) \quad\left(M_{1} \geq 8 \sqrt{2} \gamma\right)
$$

attains the global convergence $\theta(t) \rightarrow 0$, or equivalently $\boldsymbol{x}(t) \rightarrow \boldsymbol{\Psi}_{k}$, with probability one if $\theta(0) \neq \pi$.

Proof: Consider the Lyapunov function $V(\theta)=1-\cos \theta$, which satisfies $V(0)=0, V(\theta \neq 0)>0$. Noting that the function $V$ is independent of $\phi$, we have

$$
\begin{aligned}
\mathcal{L} V & =\left[u_{1}^{\prime}(t) \sqrt{\frac{1+k^{2}}{2}} \sin \frac{\theta}{2} \sin \left(\frac{\phi}{2}-\frac{\pi}{4}\right)\right. \\
& \left.-\frac{\gamma}{2} \sin \theta \cos \theta \cos ^{2} \phi\right] \frac{\partial V}{\partial \theta}+\frac{1}{2}(\sqrt{\gamma} \sin \theta \cos \phi)^{2} \frac{\partial^{2} V}{\partial \theta^{2}} \\
& =-\frac{M_{1}}{4} \sqrt{\frac{1+k^{2}}{2}} \sin ^{2} \theta(1-\sin \phi) \leq 0 .
\end{aligned}
$$

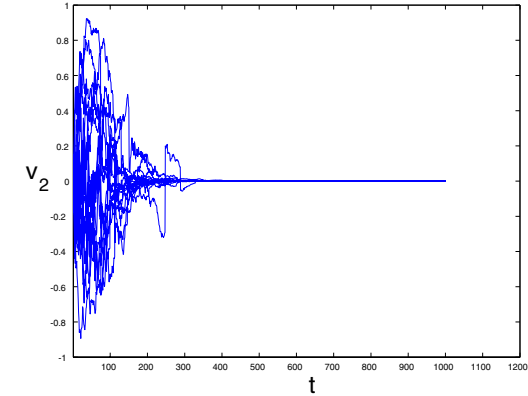

Fig. 2. Deterministic state convergence into $\boldsymbol{\Psi}_{1}$, shown by $v_{2}(\theta, \phi)$ with the initial state (12).

Hence from Lemma 1 in Appendix, $\theta=0$ is asymptotic stable. Also, from Lemma 2 we obtain $\theta \rightarrow\{0, \pi\}$ a.s., since the equality in $\mathcal{L} V \leq 0$ is attained if and only if $\theta=0, \pi$. Note that $\phi=\pi / 2$ is not an equilibrium point. We next see that the function $\tilde{V}(\theta)=-\log (1+\cos \theta)$, which satisfies $\lim _{\theta \rightarrow \pi} \tilde{V}(\theta)=+\infty$, leads to

$$
\begin{aligned}
\mathcal{L} \tilde{V} & =\frac{1}{4}(1-\cos \theta)(1-\sin \phi) \\
& \times\left[M_{1} \sqrt{\frac{1+k^{2}}{2}}-2 \gamma(1-\cos \theta)(1+\sin \phi)\right] \leq 0,
\end{aligned}
$$

where we have used the assumption $M_{1} \geq 8 \sqrt{2} \gamma$. Therefore $\mathcal{L} \tilde{V}(\theta)<0$ holds if $\theta \in(0,2 \pi) \backslash\{\pi\}$ and $\phi \in[0,2 \pi) \backslash\{\pi / 2\}$, which implies from Lemma 3

$$
\operatorname{Prob}\left[\sup _{t>0}|\theta(t)-\pi|<\pi\right]=0 \text { if } \theta(0) \in(0,2 \pi) \backslash\{\pi\} .
$$

That is, for any initial state $\theta(0) \in(0,2 \pi) \backslash\{\pi\}$ the state shows $\theta(t) \rightarrow 0$ a.s..

In the numerical simulation, we take an initial state as the non-entangled state (12), and the parameters as $\gamma=2$ and $M_{1}=32 \sqrt{2}$. Figure 2 shows the deterministic and almost global convergence into the maximally entangled state $\boldsymbol{\Psi}_{1}=\boldsymbol{\Psi}$. A remarkable fact is that this control process creates a stable maximally entangled spin pair from the non-entangled one deterministically. This entanglement creation is one of the most essential subjects in quantum information technologies. For example, the CNOT gate [7], [8], which is a well-known entangler machine in two spins, composes the quantum computer. However, the CNOT gate is a static manipulator of quantum states and does not create "stable" entangled states unlike our method.

Now we must note as well that in our formulation the excellent preparation of the maximally entangled state is only limited to the case of $k=1$, and if $k \neq 1$ we obtain the nonmaximally entangled state $\boldsymbol{\Psi}_{k}$. Therefore, we instead aim to stabilize the other maximally entangled state $\boldsymbol{\Phi}$ without respect to the initial condition. It will be shown in the next section that the above control objective is attained within the framework of approximate state preparation. The key idea is the direct use of the counterpart of Theorem 1: the DSP of $\boldsymbol{x}_{\mathrm{u}-\mathrm{u}}\left(\right.$ or $\left.\boldsymbol{x}_{\mathrm{d}-\mathrm{d}}\right)$. 


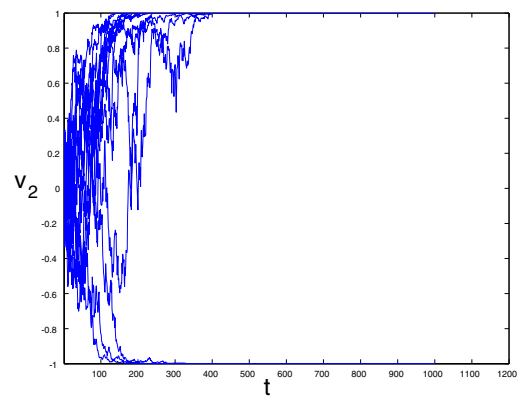

Fig. 3. State convergences into $\boldsymbol{x}_{\mathrm{u}-\mathrm{u}}$ or $\boldsymbol{x}_{\mathrm{d}-\mathrm{d}}$, shown by $v_{2}(\theta, \phi)$ with the initial state (12). The parameters are $\gamma=2$ and $M_{1}=32 \sqrt{2}$.

Proposition 2: For the controlled two-spin system (13), the feedback law

$$
u_{1}^{\prime}(t)=M_{1} \cos \frac{\theta}{2} \sin \left(\frac{\phi}{2}-\frac{\pi}{4}\right) \quad\left(M_{1} \geq 8 \sqrt{2} \gamma\right)
$$

attains the global convergence $\theta(t) \rightarrow \pi$ with probability one if $\theta(0) \neq 0$.

Proof: It is readily seen that a function $V(\theta)=1+\cos \theta$ yields $\mathcal{L} V=-M_{1} \sqrt{\left(1+k^{2}\right) / 32} \sin ^{2} \theta(1-\sin \phi) \leq 0$, which leads to $\theta \rightarrow\{0, \pi\}$ a.s.. We next observe that a function $\tilde{V}(\theta)=-\log (1+\cos \theta)$ satisfies

$$
\begin{aligned}
\mathcal{L} \tilde{V} & =\frac{1}{4}(1-\cos \theta)(1-\sin \phi) \\
& \times\left[M_{1} \sqrt{\frac{1+k^{2}}{2}}-2 \gamma(1-\cos \theta)(1+\sin \phi)\right] \leq 0 .
\end{aligned}
$$

Hence from Lemma 3, Prob[ $\left.\sup _{t>0}|\theta(t)-\pi|<\pi\right]=0$ holds if $\theta(0) \in(0,2 \pi) \backslash\{\pi\}$. Consequently, for any initial state $\theta(0) \in(0,2 \pi) \backslash\{\pi\}$ the state shows $\theta(t) \rightarrow \pi$ a.s..

After the convergence $\theta(t) \rightarrow \pi$ a.s., Eq. (15) reduces to $d \phi=-2 \gamma \sin \phi \cos \phi d t-2 \sqrt{\gamma} \sin \phi d W$. Then, by the analogous way in the above proof, it is easy to prove that this dynamics shows $\phi \rightarrow\{0, \pi\}$ a.s., or equivalently, $\boldsymbol{x}(t) \rightarrow\left\{\boldsymbol{x}_{\mathrm{u}-\mathrm{u}}, \boldsymbol{x}_{\mathrm{d}-\mathrm{d}}\right\}$ a.s., which is shown in Fig.3.

\section{Approximate preparation of the maximally entangled state}

In this subsection, we aim to stabilize the other maximally entangled state (4) without respect to the value of $k$. The representation of $\boldsymbol{\Phi}$ in the two-dimensional coordinate is given by $(\theta, \phi)=(\pi, \pi / 2)$. Note that the strict convergence into $\boldsymbol{\Phi}$ is impossible because $(\theta, \phi)=(\pi, \pi / 2)$ is not an equilibrium point of the dynamics. Therefore, the control objective will be achieved in the following sense: the expectation value of a function that represents the distance from $\boldsymbol{\Phi}$ can be made arbitrarily small by tuning the control gain.

We now note that a part of the above control subject has already been attained; for the dynamics (13), the feedback control law (17) leads to the almost global convergence $\theta(t) \rightarrow \pi$ with probability one. Hence, we can focus on the control of the $\phi$-variable by switching the Hamiltonian from $H_{1}$ to $H_{2}$ after the convergence. Then, the controlled dynamics (15) is reduced to

$$
d \phi=\left[u_{2}-2 \gamma \sin \phi \cos \phi\right] d t-2 \sqrt{\gamma} \sin \phi d W .
$$

Then, the stabilizing controller is given in the following theorem.

Theorem 3: For the dynamics (18) on $\phi \in[0,2 \pi)$, consider the feedback law

$$
u_{2}(t)=-M \sin \left(\frac{\phi(t)}{2}-\frac{\pi}{4}\right),
$$

which is discontinuous at $\phi=0(\sim 2 \pi)$. Then, the positive semidefinite function $V(\phi)=1-\cos (\phi / 2-\pi / 4)$ satisfies

$$
\mathbf{E}[V(\phi(t))] \leq V(\phi(0)) \mathrm{e}^{-\bar{M} t}+\frac{6 \gamma}{(2-\sqrt{2}) M},
$$

where $\bar{M}:=(2-\sqrt{2}) M / 4$.

Proof: When we take $\phi^{\prime}=\phi-\pi / 2$ for simplicity, the derivative of the Lyapunov function $V\left(\phi^{\prime}\right)=1-\cos \left(\phi^{\prime} / 2\right)$ becomes

$$
\begin{aligned}
\mathcal{L} V= & {\left[u_{2}(t)+2 \gamma \sin \phi^{\prime} \cos \phi^{\prime}\right] \frac{1}{2} \sin \frac{\phi^{\prime}}{2} } \\
& +\frac{1}{2}\left(-2 \sqrt{\gamma} \cos \phi^{\prime}\right)^{2} \frac{1}{4} \cos \frac{\phi^{\prime}}{2} \\
< & -\frac{M}{2} \sin ^{2} \frac{\phi^{\prime}}{2}+\gamma+\frac{\gamma}{2} \\
= & -\frac{M}{2}\left(1+\cos \frac{\phi^{\prime}}{2}\right)\left(1-\cos \frac{\phi^{\prime}}{2}\right)+\frac{3 \gamma}{2}
\end{aligned}
$$

Now due to $-\pi / 2 \leq \phi^{\prime}<3 \pi / 2$ we have $(2-\sqrt{2}) / 2<$ $1+\cos \left(\phi^{\prime} / 2\right)$, which leads to

$$
\begin{aligned}
\mathcal{L} V & <-\left(\frac{2-\sqrt{2}}{4} M\right)\left(1-\cos \frac{\phi^{\prime}}{2}\right)+\frac{3 \gamma}{2} \\
& =-\left(\frac{2-\sqrt{2}}{4} M\right) V+\frac{3 \gamma}{2} .
\end{aligned}
$$

Therefore from Lemma 4 we have the assertion.

Clearly, the feedback controller with a large gain $M \gg 1$ makes the expectation of the distance function, $\mathbf{E}[V(\phi)]$, close to zero as $t \rightarrow \infty$. This implies that we can stabilize $\phi(t)$ globally and approximately at $\phi=\pi / 2$. Let us see the degree of the approximation by a simulation. Take the parameters as $\gamma=2, M=32$ and the initial condition as $\phi(0)=\pi / 2$. A standard criterion which measures the distance between two quantum states $\boldsymbol{x}_{1}$ and $\boldsymbol{x}_{2}$ is the fidelity: $f:=\left|\boldsymbol{x}_{1}^{*} \boldsymbol{x}_{2}\right|^{2}$, which is bounded by $0 \leq f \leq 1$. The fidelity becomes 1 if and only if $\boldsymbol{x}_{1}=\boldsymbol{x}_{2}$. Now the fidelity between the maximally entangled state $\boldsymbol{\Phi}$ and our quantum state $\boldsymbol{x}(\pi, \phi)$ is given by

$$
f=\left|\boldsymbol{x}(\pi, \phi)^{T} \boldsymbol{\Phi}\right|^{2}=\frac{1}{2}(1+\sin \phi(t)) .
$$

Figure 5 shows the time-dependence of the fidelity $f(t)$, where we can see that the noise effect at $\phi=\pi / 2$ is well suppressed, and the approximate state preparation of $\boldsymbol{\Phi}$ is realized. 


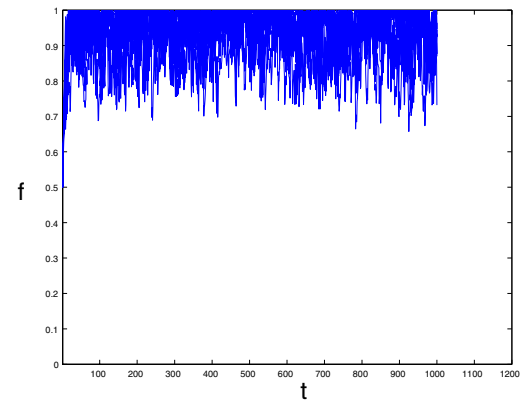

Fig. 4. Time-dependence of the fi delity between the maximally entangled state $\boldsymbol{\Phi}$ and our quantum state $\boldsymbol{x}(\pi, \phi)$.

\section{CONCLUSION}

We have considered some feedback control problems of the two-spin system subject to the perfect continuous measurement. The dynamics has four equilibrium points corresponding to the up-up, the down-down, and the mixing of up-down (duplication) state, the last of which has a special quantum correlation between spins, i.e., the entanglement.

Firstly, under the assumption that the initial state takes a real vector, we have derived a two-dimensional description of the dynamics, which makes some useful formulas in stochastic control theory directly applicable. Then, it was proved that a feedback controller stabilizes the abovementioned entangled state almost globally and deterministically. Moreover, we have provided a feedback controller stabilizing the other entangled state, which corresponds to a non-equilibrium point of the dynamics, in the sense that the expectation value of the distance from the target state can be made arbitrarily small by tuning the control gain.

All the facts obtained in this paper demonstrates that the feedback control set-up is highly expected to be a robust entangler machine for various kinds of quantum systems. In particular, we now obtain a new conjecture that some type of Hamiltonians have a possibility to achieve the global and approximate preparation of a wide class of entangled states, in addition to those corresponding to the eigenvectors of the measured observable. For the future investigations, we have to proceed into control problems of the multi-spin system without assuming the perfect measurement, in which case some computational approaches will be necessary.

\section{APPENDIX}

Consider an Ito $\mathrm{SDE}$ on $\mathbf{R}^{n}$ of the form

$$
d x=f(x) d t+g(x) d W,
$$

where $f(x)$ and $g(x)$ are smooth vector fields. Suppose that the system has an equilibrium point $x_{o}$ satisfying $f\left(x_{o}\right)=$ $g\left(x_{o}\right)=0$. Define the infinitesimal generator of $x$ as

$$
\mathcal{L}:=\sum_{i} f_{i} \frac{\partial}{\partial x_{i}}+\frac{1}{2} \sum_{i, j}\left(g g^{T}\right)_{i j} \frac{\partial^{2}}{\partial x_{i} \partial x_{j}} .
$$

Then, the following lemmas [16], [17] hold.

Lemma 1: Consider a ball $U_{r}=\left\{x|| x-x_{o} \mid<r\right\}$.
Suppose there exists a function $V: U_{r} \rightarrow \mathbf{R}_{+}$such that $V\left(x_{o}\right)=0$ and $V(x)>0$ otherwise, and $\mathcal{L} V(x) \leq 0$ on $U_{r}$. Then the equilibrium point $x_{o}$ is stable in probability, i.e.,

$$
\lim _{x \rightarrow x_{o}} \operatorname{Prob}\left[\sup _{t \geq 0}\left|x-x_{o}\right|>\epsilon\right]=0 .
$$

Lemma 2: Let $G$ be a bounded invariant set with respect to the solution of $(21)$ and $x(0) \in G$. Suppose there exists a function $V: G \rightarrow \mathbf{R}_{+}$such that $\mathcal{L} V(x) \leq 0$ holds for all $x \in G$. Then we have $\lim _{t \rightarrow \infty} \mathcal{L} V(x)=0$ a.s.

Lemma 3: Suppose there exists a function $V: U_{r} \rightarrow$ $\mathbf{R}$ such that $\lim _{x \rightarrow x_{o}} V(x)=+\infty$ and $\mathcal{L} V(x)<0$ on $U_{r} \backslash\left\{x_{o}\right\}$. Then we have

$$
\operatorname{Prob}\left[\sup _{t>0}\left|x-x_{o}\right|<r\right]=0, \quad \forall x(0) \in U_{r} \backslash\left\{x_{o}\right\} .
$$

Lemma 4: Suppose there exist a $C^{2}$ function $V(x) \geq 0$, constants $\alpha>0$ and $\beta>0$ such that $\mathcal{L} V(x) \leq-\alpha V(x)+\beta$ holds for all $x \in \mathbf{R}^{n}$ and $t \geq 0$. Then, there is a unique solution of (21) for each $x(0)$ and it satisfies

$$
\mathbf{E}[V(x(t))] \leq V(x(0)) \mathrm{e}^{-\alpha t}+\beta / \alpha .
$$

\section{REFERENCES}

[1] V. P. Belavkin: Quantum stochastic calculus and quantum nonlinear fi ltering, J. Multivariate Anal., vol.42, pp.171-201 (1992).

[2] C. M. Caves and G. J. Milburn: Quantum-mechanical model for continuous position measurements, Phys. Rev. A36, 5543 (1987).

[3] L. Bouten, M. Guta and H. Maassen: Stochastic Schrödinger equations, J. Phys. A, vol.37, pp.3189-3209 (2004).

[4] A. C. Doherty, S. Habib, H. Mabuchi and K. Jacobs: Quantum feedback control and classical control theory, Phys. Rev. A62, 012105 (2000).

[5] J. Wang and H. M. Wiseman: Feedback stabilization of an arbitrary pure state of a two-level atom, Phys. Rev. A64, 063810 (2001).

[6] C. Ahn, A. C. Doherty and A. J. Landahl: Continuous quantum error correction via quantum feedback control, Phys. Rev. A65, 042301 (2002).

[7] P. Shor, Scheme for reducing decoherence in quantum computer memory, Phys. Rev. A52, 2493 (1995).

[8] M. A. Nielsen and I. L. Chuang: Quantum Computation and Quantum Information Cambridge University Press, Cambridge (2000).

[9] C. H. Bennet, et al, Teleporting an unknown quantum state via dual classical and Einstein-Podolsky-Rosen channels, Phys. Rev. Lett.70, 1895 (1993).

[10] J. K. Stockton, R. van Handel and H. Mabuchi: Deterministic Dicke state preparation with continuous measurement and control, Phys. Rev. A70, 022106 (2004).

[11] R. van Handel, J. K. Stockton and H. Mabuchi: Feedback control of quantum state reduction, submitted to IEEE Trans. Automat. Contr.

[12] D. P. DiVincenzo: Two-bit gates are universal for quantum computation, Phys. Rev. A50, 1015 (1995).

[13] C. W. Gardiner: Handbook of Stochastic Methods, Springer, Berlin (1983).

[14] R. P. Feynman, R. B. Leighton and M. L. Sands, The Feynman Lecture Note on Physics, vol.III Addison-Wesley, 1965.

[15] M. Kitagawa and M. Ueda: Squeezed spin states, Phys. Rev. A47, 5138 (1993).

[16] R. Z. Has'minskii, Stochastic stability of differential equations, Sijthoff \& Noordhoff (1980).

[17] H. Deng, M. Krstic and R. J. Williams, Stabilization of stochastic nonlinear systems driven by noise of unknown covariance, IEEE Trans. Automat. Contr., vol.46, pp.1237-1253, 2001. 